\title{
ESTUDO ECONÔMICO DE VIABILIDADE PARA AQUISIÇÃO DE UM TOMÓGRAFO COMPUTADORIZADO POR UM ESTABELECIMENTO DE APOIO À SAÚDE FILANTRÓPICO ${ }^{1}$
}

\author{
FEASIBILITY STUDY FOR ACQUISITION OF A COMPUTED \\ TOMOGRAPHY BY A PHILANTHROPIC HEALTH SUPPORT ESTABLISHMENT
}

\author{
Leandro Welter Caetano², Jaqueline Carla Guse ${ }^{3}$ e Joana Bratz Lourenço ${ }^{4}$
}

\section{RESUMO}

Este estudo apresenta a viabilidade econômica para a aquisição de um Tomógrafo Computadorizado para um Estabelecimento Assistencial de Saúde (EAS) da cidade de Santa Maria, Rio Grande do Sul. O EAS é uma entidade privada e filantrópica e seu centro de radiodiagnóstico é de pequeno porte, sendo que $88,64 \%$ de seus exames de imagem são realizados para convênios com o Sistema Único de Saúde (SUS). Foram utilizados os modelos do Valor Presente Líquido (VPL) e Taxa Interna de Retorno (TIR) para a avaliação de viabilidade. Com base na previsão de ampliação do EAS, estimou-se três cenários, um pessimista, um esperado e um otimista, para o período de 2020 a 2024. Um levantamento dos dados da unidade do período de um ano foi realizado para avaliar as demandas e definições com a equipe de gestão do hospital especificando os parâmetros e critérios adotados neste estudo, que demonstra a importância da avaliação de um empreendimento para a tomada de decisões.

Palavras-chave: Engenharia Econômica, Radiodiagnóstico, Exames médicos, Análise de Cenários.

\section{ABSTRACT}

This work shows the economic viability to the acquisition of computed tomography to a Health Care Establishment (HCE) from the Santa Maria city, Rio Grande do Sul. The HCE is a private and philanthropic entity and its radiodiagnosis center is small, with $88.64 \%$ of its imaging exams performed through agreements with the Unified Health System (SUS). The Liquid Present Value (LPV) and Internal Rate of Return (IRR) were used to evaluate the viability. Considering the ampliation prevision of the HCE, three scenarios were estimated, a pessimist, an expected, and an optimistic, to the period of 2020 to 2024. A survey of the data from the unit for one year was carried out to evaluate the demands and definitions with the hospital management team specifying the parameters and criteria adopted in this study, which demonstrates the importance of evaluating an enterprise for decision making.

Keywords: Economic Engineering, Radiodiagnosis, Medical examinations, Scenario Analysis.

\footnotetext{
${ }^{1}$ Trabalho de Conclusão de curso.

${ }^{2}$ Acadêmico do Curso de Engenharia Biomédica - Universidade Franciscana - UFN. E-mail: leandro.caetano@gmail.com

${ }^{3}$ Professora do Curso de Ciências Contábeis - Universidade Franciscana - UFN. E-mail: jaqueline_guse@hotmail.com

${ }^{4}$ Orientadora - Professora do curso de Engenharia de Materiais - Universidade Franciscana - UFN. E-mail: joana.lourenco@ ufn.edu.br
} 


\section{INTRODUÇÃO}

Um estudo de viabilidade econômica, envolve uma série de análises sobre o mercado com o intuito de entender se o projeto é viável para ser executado (GREER e KOLBE, 2003). Para uma completa análise econômica e financeira de um empreendimento devem ser avaliadas todas as quantidades envolvidas, sejam elas entradas ou saídas de caixa (GOLDMAN, 2015).

O estudo de viabilidade econômica é realizado a partir da análise dos Fluxos de Caixa (FC), o qual pode ser entendido como uma sucessão de recebimentos $\left(\mathrm{R}_{\mathrm{t}}\right)$ ou de pagamentos $\left(\mathrm{C}_{\mathrm{t}}\right)$ previsto para determinado período de tempo (n) representado por um diagrama (Figura 1) (VIEIRA SOBRINHO, 2015).

A separação dos gastos em custos e despesas é fundamental para a apuração do custo da produção e do resultado de um período. Salienta-se que custos são gastos necessários para a produção de bens e serviços, enquanto despesas são gastos necessários para geração de receitas (PEREZ JR, DE OLIVEIRA e COSTA, 2011).

Figura 1 - Diagrama de Fluxo de Caixa

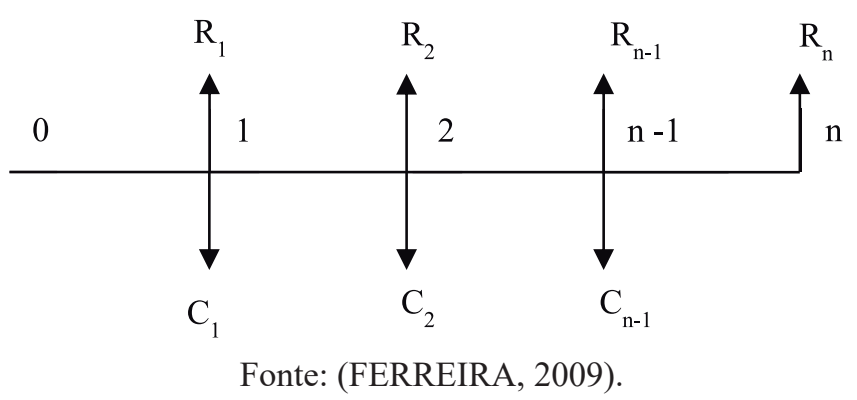

Para avaliar a viabilidade de um empreendimento, os fluxos de caixa podem ser avaliados a partir de indicadores como o Valor Presente Líquido (VPL) e a Taxa Interna de Retorno (TIR).

O VPL é a diferença entre o valor investido $\left(\mathrm{CF}_{0}\right)$ e o valor dos benefícios esperados $\left(\mathrm{CF}_{\mathrm{j}}\right)$, descontados para a data inicial, usando-se como taxa de desconto a Taxa Mínima de Atratividade (TMA) (CLEMENTE, 2008).

A TMA é a taxa a partir da qual o investidor está obtendo ganhos financeiros, associada a um baixo risco e alta liquidez. Ao se analisar uma proposta de investimento deve ser considerado o fato de estar perdendo a oportunidade de auferir retornos pela aplicação do mesmo capital em outros projetos (CASAROTO FILHO, 2010). Quando o VPL for positivo, o projeto é capaz de recuperar o investimento inicial, podendo-se considerar o projeto, do contrário, ele não é viável (OLIVIO, 2013). A Equação 1 representa o cálculo deste valor, onde I é o investimento e FC são os fluxos de caixa do projeto.

$$
V P L=-I+\sum F C
$$


A TIR de um projeto pode ser interpretada do ponto de vista matemático como a taxa que torna nulo o VPL de um fluxo de caixa (CLEMENTE, 2008). Observa-se, assim que, ao invés de buscarmos o resultado de valores descontados, como no VPL, na TIR buscamos a taxa de juros, tornando-a uma incógnita (OLIVIO, 2013). A TIR consiste em considerá-la como um valor limite da TMA, isto é, o projeto será considerado viável enquanto sua TIR for maior que a TMA.

Como complemento ao estudo de viabilidade, é analisado o comportamento da rentabilidade do projeto por meio da Análise de Cenários e da Análise de Sensibilidade (OLIVIO, 2013).

A Análise de Sensibilidade é utilizada para o caso de alguns componentes do fluxo de caixa que estão sujeitos a certa margem de imprevisibilidade. Para desenvolver a análise de sensibilidade, é necessário variar os parâmetros de entrada, resolver o problema e registrar os resultados obtidos. Para cada parâmetro utilizado, haverá uma variação dos indicadores econômicos. Os parâmetros que, proporcionalmente, provocarem maior variação nestes indicadores são classificados como sensíveis (CLEMENTE, 2008).

$\mathrm{Na}$ análise de cenários devem ser concebidos simultaneamente três cenários futuros potenciais que estimulem a percepção de diferentes possibilidades, dos quais se possam tirar respostas adequadas, sendo estes cenários pessimista, esperado e otimista (SERTEK, GUINDANI e MARTINS, 2007).

Outro aspecto importante a ser levado em conta nos cálculos de viabilidade é a depreciação, a qual é a diminuição do valor de um bem resultante do desgaste pelo uso, ação da natureza ou obsolência normal, podendo ser real ou contábil. A depreciação real é a diminuição efetiva do valor de um bem resultante do desgaste pelo uso, ação da natureza ou obsolência normal. A depreciação contábil é a diminuição do valor contábil de um bem, resultante do decurso do prazo decorrido desde a sua aquisição até o instante atribuído ao desgaste físico, ao uso ou à obsolência (HIRSCHFELD, 2000). A ideia básica associada a constituição de um fundo de depreciação é a de permitir que, ao se dar baixa em um bem depreciável, o valor monetário correspondente acumulado nesse fundo seja suficiente para a aquisição de outro bem similar (CASAROTO FILHO, 2010).

Sendo assim, no presente trabalho será apresentado um estudo de viabilidade da compra de um tomógrafo para ser instalado em um Estabelecimento Assistencial de Saúde (EAS), entidade privada, sem fins lucrativos, localizada na cidade de Santa Maria, no estado do Rio Grande do Sul. Como critério de avaliação da viabilidade, serão considerados o VPL e a TIR, para três cenários, pessimista, esperado e otimista, para um período de cinco anos, de 2020 a 2024.

\section{MATERIAIS E MÉTODOS}

O EAS, objetivo deste estudo, possui um setor de Serviços de Radiodiagnóstico que atende as demandas de exames particulares, por convênios e pelo Sistema Único de Saúde (SUS) com origem nos hospitais e postos de saúde municipais de Santa Maria e outros municípios da região central, bem como demandas das unidades de pronto socorros e urgência. 
O setor de radiodiagnóstico conta com um equipamento de tomografia computadorizada, porém é antigo e necessita constantemente de paradas para manutenção, causando insegurança em sua confiabilidade e disponibilidade operacional, por isso se faz necessária a aquisição de um novo equipamento, que garanta maior disponibilidade e confiabilidade.

Foram apresentadas soluções e respectivas propostas de equipamentos de Tomografia Computadorizada por empresas que vendem equipamentos médicos, que atendam as características deste EAS. Dentre as propostas apresentadas, a equipe técnica do EAS escolheu o equipamento que melhor atendia aos requisitos técnicos e operacionais para o serviço de Radiodiagnóstico deste EAS, para o qual será realizado o presente estudo de viabilidade.

O estudo levou em consideração o período de agosto de 2018 a agosto de 2019 para prever o número de exames realizados na unidade. Este estudo leva em conta uma projeção de 5 anos, de 2020 a 2024 para o cálculo da TIR e do VPL.

\section{PROJETO E INVESTIMENTOS INICIAIS}

O projeto em estudo faz uma projeção econômica de cinco anos e constitui-se na aquisição e instalação de um tomógrafo computadorizado. Foram considerados para o investimento inicial depreciável o tomógrafo computadorizado, a mobília, o ar condicionado e o desumidificador de ar. Com relação aos custos não depreciáveis, foram consideradas as obras civis e adequações elétricas.

\section{ESTIMATIVAS DE GASTOS OPERACIONAIS}

Foram levantadas, juntamente com a gerência do setor de Serviço de Rádiodiagnóstico, as principais variáveis que compõe as saídas de caixa, custos e despesas, fixos e variáveis, relacionados a operação do equipamento e locação das áreas necessárias para seu funcionamento, os quais são apresentados na Tabela 1 .

Um percentual de 4,0\% ao ano deve ser considerado, sobre o faturamento bruto, para gastos com manutenção do equipamento de tomografia, conforme a média nacional (TELES, 2019). No entanto, este valor só será considerado a partir do segundo ano, pois o fornecedor do equipamento concede garantia de um ano.

Além disso, será considerado um valor de 4,95 \% ao ano de aumento dos custos fixos totais, sendo esta taxa relativa ao Índice Geral de Preço do Mercado - IGP-M, definido para o período de agosto de 2018 a agosto de 2019 (FGV, 2019). 
Tabela 1 - Saídas de caixa mensal do Serviço de Rádiodiagnóstico.

\begin{tabular}{lccccc}
\hline \multicolumn{1}{c}{ Item } & Valor unitário & Quantidade & Unidade & Total receita & Tipo \\
\hline Equipe técnica Radiologia & $\mathrm{R} \$ 4.605,56$ & 2 & Pessoa/mês & $\mathrm{R} \$ 9.211,11$ & Fixo \\
\hline Equipe técnica Enfermagem & $\mathrm{R} \$ 2.589,56$ & 1 & Pessoa/mês & $\mathrm{R} \$ 2.589,56$ & Fixo \\
\hline Higienização & $\mathrm{R} \$ 500,00$ & 1 & mês & $\mathrm{R} \$ 500,00$ & Fixo \\
\hline Física Médica & $\mathrm{R} \$ 800,00$ & 1 & mês & $\mathrm{R} \$ 800,00$ & Fixo \\
Software ANIMATI & $\mathrm{R} \$ 350,00$ & 1 & mês & $\mathrm{R} \$ 350,00$ & Fixo \\
\hline Energia Elétrica & $\mathrm{R} \$ 2.000,00$ & 1 & mês & $\mathrm{R} \$ 2.000,00$ & Fixo \\
\hline Locação da área para Tomógrafo & $\mathrm{R} \$ 1.250,00$ & 1 & mês & $\mathrm{R} \$ 1.250,00$ & Fixo \\
\hline Digitação & $\mathrm{R} \$ 3,00$ & 1 & unitário & $\mathrm{R} \$ 3,00$ & Variável \\
\hline Laudo & $\mathrm{R} \$ 30,00$ & 1 & unitário & $\mathrm{R} \$ 30,00$ & Variável \\
\hline Recepção e impressão & $\mathrm{R} \$ 0,80$ & 1 & unitário & $\mathrm{R} \$ 0,80$ & Variável \\
\hline
\end{tabular}

Fonte: Construção do autor

\section{ESTIMATIVAS DE RECEITAS}

Com o intuito de conhecer o perfil atual das entradas de receita, foi realizada uma pesquisa para levantar o histórico no período de um ano, de agosto de 2018 a agosto de 2019, nos exames realizados, sendo os dados apresentados na Tabela 2.

Do total de exames realizados, Abdome, Coluna lombar, Tórax e Crânio somam 91,24\%; dentre todos os exames, os realizados pelo SUS representarem $88,64 \%$ do total, sendo os demais atendidos por convênios médicos e pagamentos particulares.

Tabela 2 - Exames realizados de agosto de 2018 a agosto de 2019

\begin{tabular}{|lcc}
\hline \multicolumn{1}{c}{ Exame } & Número de exames & Percentual \\
\hline Sela túrcica & 01 & $0,03 \%$ \\
\hline Pelve & 09 & $0,28 \%$ \\
\hline Abdome inferior & 10 & $0,31 \%$ \\
\hline Angiotomografia & 14 & $0,43 \%$ \\
\hline Coluna torácica & 17 & $0,52 \%$ \\
\hline Coluna dorsal & 19 & $0,58 \%$ \\
\hline Abdome superior & 39 & $1,19 \%$ \\
\hline Face & 51 & $1,56 \%$ \\
\hline Pescoço & 57 & $1,75 \%$ \\
\hline Coluna cervical & 69 & $2,11 \%$ \\
\hline Abdome & 367 & $11,24 \%$ \\
\hline Coluna lombar & 380 & $11,64 \%$ \\
\hline Tórax & 500 & $15,31 \%$ \\
\hline Crânio & 1732 & $53,05 \%$ \\
\hline Total & 3265 & $100,00 \%$ \\
\hline & Fonte: Construção do autor & \\
\hline
\end{tabular}

Devido à dificuldade de acesso aos valores atualizados de todos os convênios disponíveis, neste estudo optou-se por fazer a análise da viabilidade considerando apenas estes quatro principais 
exames realizados (Abdome, Coluna lombar, Tórax e Crânio), onde utilizou-se valores dos exames definidos pela tabela SUS (DATASUS, 2019). Desta forma, uma média ponderada por exame foi estabelecida no valor de R\$99,93, conforme apresentado na Tabela 3, o qual será utilizado como base de cálculo para o valor de cada exame.

Tabela 3 - Custo médio ponderado considerado para este estudo

\begin{tabular}{lcc}
\hline \multicolumn{1}{c}{ Exame } & Percentual $^{*}$ & Valor unitário $^{* * *}$ \\
\hline Abdome & $15,59 \%$ & $\mathrm{R} \$ 138,63$ \\
Coluna Lombar & $11,78 \%$ & $\mathrm{R} \$ 101,10$ \\
Tórax & $20,90 \%$ & $\mathrm{R} \$ 136,41$ \\
Crânio & $51,73 \%$ & $\mathrm{R} \$ 97,44$ \\
Total & $100 \%$ & $\mathrm{R} \$ 99,93^{* * *}$ \\
\hline
\end{tabular}

Fonte: Construção do autor

${ }^{*}$ Dados coletados na EAS de agosto de 2018 a agosto de 2019; **(DATASUS, 2019);

${ }^{* * *}$ Valor de receita média ponderada entre os exames para o período do presente estudo

\section{CUSTO DE CAPITAL, IMPOSTOS E DEPRECIAÇÃO}

Para calcular o VPL, adotou-se para a Taxa Mínima de Atratividade (TMA) a Taxa do Sistema Especial de Liquidação e Custódia (SELIC) fornecida pelo Banco Central do Brasil. A taxa SELIC representa os juros básicos da economia brasileira, um sistema administrado pelo Banco Central em que são negociados títulos públicos federais. A SELIC é um dos elementos centrais da estratégia de política monetária no Brasil, que está baseada em um sistema de metas de inflação (MISHKIN, 2000).

Para agosto de 2019, seu valor foi de 5\% ao ano, e com projeção de 5\% ao ano para 2020 e 7\% ao ano para 2021 e 2022 (BANCO CENTRAL DO BRASIL, 2019), portanto, uma média de 6\% ao ano será considerada neste estudo para a Taxa Mínima de Atratividade.

A EAS é uma instituição filantrópica, que possui imunidade tributária de acordo com o Artigo 150 da Constituição Federal de 1988 e, portanto, não paga Imposto de Renda:

\footnotetext{
Art. 150 - Sem prejuízo de outras garantias asseguradas ao contribuinte, é vedado à união, aos estados, ao distrito federal e aos municípios:

(...)

vi - instituir impostos sobre:

(...)

c) patrimônio, renda ou serviços dos partidos políticos, inclusive suas fundações, das entidades sindicais dos trabalhadores, das instituições de educação e de assistência social, sem fins lucrativos, atendidos os requisitos da lei. (BRASIL, 2020)
}

Uma taxa de depreciação de $10 \%$ será considerada para o mobiliário e os equipamentos adquiridos pelo hospital, conforme a Instrução Normativa RFB nº 1700, de 14 de março de 2017 (BRASIL, 2017). 
Para a análise de sensibilidade foram realizadas modificações nas variáveis, a fim de observar quais destas mais influenciam no VPL. Identificou-se que quantidade e valor dos exames são as variáveis que mais influenciam no resultado do projeto, portanto as que sofrerão variação nas composições de cenários. Como a tabela SUS (DATASUS, 2019) não sofre alterações desde 2007, para a análise de sensibilidade será considerado como critério apenas um aumento do número de exames, definido em conjunto com a EAS.

O cenário esperado é de um aumento de $8 \%$ ao ano para o primeiro e segundos anos, $10 \%$ ao ano para o terceiro e quartos anos e 15\% ao ano para o quinto ano. Para a análise de cenários, considerou que para o cenário pessimista haveria uma diminuição de $50 \%$ número de exames e no cenário otimista, um aumento de 50\%, ambos em relação ao cenário esperado, conforme apresentado na Tabela 4.

Tabela 4 - Análises de cenários com relação à expectativa de aumento do número de exames

\begin{tabular}{cccccc}
\hline \multirow{2}{*}{ Cenário } & $\mathbf{5}$ & Período (anos) \\
\cline { 2 - 6 } & $\mathbf{1}$ & $\mathbf{2}$ & $\mathbf{3}$ & $\mathbf{4}$ & $\mathbf{5}$ \\
\hline Esperado & $+8 \%$ & $+8 \%$ & $+10 \%$ & $+10 \%$ & $+15 \%$ \\
Otimista & $+12 \%$ & $+12 \%$ & $+15 \%$ & $+15 \%$ & $+22,5 \%$ \\
Pessimista & $+4 \%$ & $+4 \%$ & $+5 \%$ & $+5 \%$ & $+7,5 \%$ \\
\hline
\end{tabular}

Fonte: Construção do autor

\section{RESULTADOS E DISCUSSÕES}

Os fluxos de caixas para os três cenários são apresentados nas Figuras 2 a 4. Considerando uma depreciação de $10 \%$ ao ano, ao longo de 5 anos, para os investimentos depreciáveis, o valor de R\$ 505.000,00 é referente ao valor residual contábil ao final deste período. Como não há incidência de imposto de renda sobre este valor, ele foi trazido para o presente como lucro para o ano zero.

Figura 2 - Fluxo de caixa Pessimista

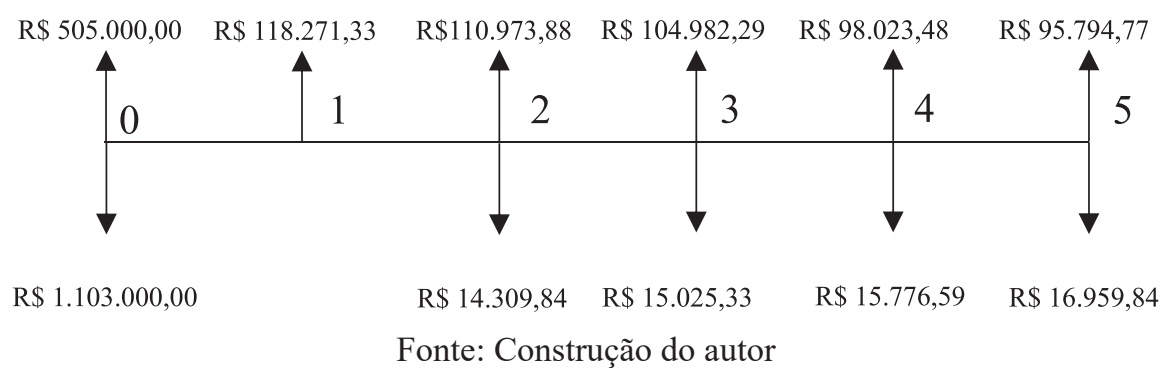


Figura 3 - Fluxo de caixa Esperado

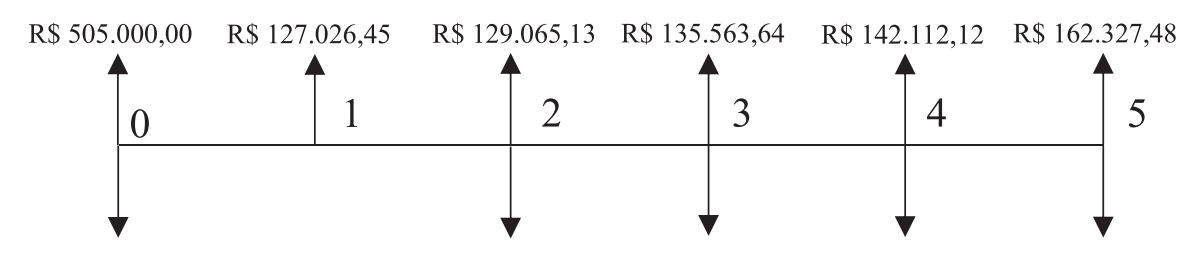

$\begin{array}{lllll}\mathrm{R} \$ 1.103 .000,00 & \mathrm{R} \$ 15.431,76 & \mathrm{R} \$ 16.974,94 & \mathrm{R} \$ 18.672,43 & \mathrm{R} \$ 21.473,30\end{array}$

Fonte: Construção do autor

Figura 4 - Fluxo de caixa Otimista

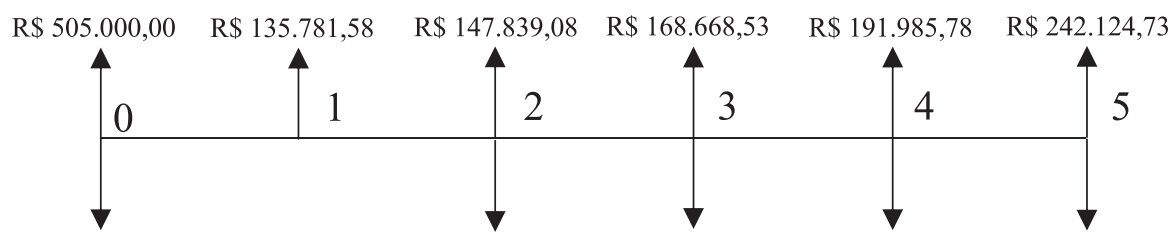

R\$ $1.103 .000,00$

$\mathrm{R} \$ 16.596,02 \quad \mathrm{R} \$ 19.085,43 \quad \mathrm{R} \$ 21.948,24 \quad \mathrm{R} \$ 26.886,59$

Fonte: Construção do autor

Na Tabela 5 são apresentadas as estimativas de Valor Presente Líquido (VPL) e Taxa Interna de Retorno (TIR) para os três cenários avaliados neste estudo, pessimista, esperado e otimista. Verifica-se que para a avaliação suposta neste estudo, considerando o VPL, apenas no cenário otimista o investimento é viável, apresentando um valor positivo. No entanto, considerando a TIR, seu valor de $4,11 \%$ é menor que a TMA, considerada em $6 \%$ ao ano, o que significa que o investimento seria inviável. Portanto, a menos que o valor da Taxa SELIC venha a diminuir ao longo dos cinco anos considerados para a análise, o investimento não é viável.

Tabela 5 - Estimativas de Valor Presente Líquido e Taxa Interna de Retorno para os três cenários.

\begin{tabular}{lccc}
\hline \multicolumn{1}{c}{ Definição } & Pessimista & Esperado & Otimista \\
\hline Taxa Mínima de Atratividade - SELIC & $6.00 \%$ & $6.00 \%$ & $6.00 \%$ \\
\hline Valor Presente Investimentos & $\mathrm{R} \$ 1,103,000.00$ & $\mathrm{R} \$ 1,103,000.00$ & $\mathrm{R} \$ 1,103,000.00$ \\
\hline Valor Presente Incremental & $\mathrm{R} \$ 50,521.18$ & $\mathrm{R} \$ 58,823.11$ & $\mathrm{R} \$ 68,271.18$ \\
\hline Valor Presente Investimento Total & $-\mathrm{R} \$ 1,153,521.18$ & $-\mathrm{R} \$ 1,161,823.11$ & $-\mathrm{R} \$ 1,171,271.18$ \\
Valor Presente (Fluxo de Caixa Livre) & $\mathrm{R} \$ 447,715.44$ & $\mathrm{R} \$ 582,392.28$ & $\mathrm{R} \$ 734,289.83$ \\
\hline Valor residual do equipamento & $\mathrm{R} \$ 505,000.00$ & $\mathrm{R} \$ 505,000.00$ & $\mathrm{R} \$ 505,000.00$ \\
\hline Valor Presenta Líquido - VPL & $-\mathrm{R} \$ 705,805.74$ & $-\mathrm{R} \$ 74,430.83$ & $\mathrm{R} \$ 68,018.65$ \\
\hline Taxa Interna de Retorno - TIR & $-11.64 \%$ & $-4.40 \%$ & $4.11 \%$ \\
\hline
\end{tabular}

Fonte: Construção do autor

Outro ponto levado em consideração foi a capacidade da unidade, a qual foi levantada considerando-se atendimentos durante 8 horas por dia, ao longo de 5 dias por semana, com cada exame levando em média $20 \mathrm{~min}$, de forma que ao longo de um ano o tomógrafo consegue fazer 5760 exames.

Na Tabela 6 a projeção de exames realizada para os cenários definidos neste estudo é apresentada. Verifica-se que para o quinto ano do cenário otimista, o número de exames projetados passa da 
capacidade da máquina e, portanto, deve-se pensar em alternativas para aumentar a capacidade da unidade a partir deste período.

Tabela 6 - Projeção de exames realizados por ano

\begin{tabular}{lccccc}
\hline & \multicolumn{5}{c}{ Período (anos) } \\
\cline { 2 - 6 } & $\mathbf{1}$ & $\mathbf{2}$ & $\mathbf{3}$ & $\mathbf{4}$ & $\mathbf{5}$ \\
\hline Número de exames - Cenário pessimista & 3442 & 3580 & 3759 & 3947 & 4243 \\
Número de exames - Cenário esperado & 3575 & 3861 & 4247 & 4672 & 5372 \\
Número de exames - Cenário otimista & 3707 & 4152 & 4775 & 5491 & $6727^{*}$ \\
\hline Fonte: Construção do autor \\
*Valor acima da capacidade projetada de 5760 exames.
\end{tabular}

Para que a demanda não exceda a capacidade de exames, pode-se considerar que os exames possam ser realizados aos sábados ou pode-se ainda melhorar a eficiência da gestão do tempo disponibilizado para cada exame, hoje em 20 min, decisões que devem ser discutidas com os gestores da EAS.

\section{CONCLUSÃO}

Um estudo econômico de viabilidade para aquisição de um tomógrafo computadorizado por um estabelecimento de apoio à saúde filantrópico foi apresentado. Verifica-se que a avaliação da viabilidade de um investimento é fator decisivo para o sucesso de um empreendimento e seu planejamento evita que decisões erradas sejam tomadas.

O Valor Presente Líquido (VPL) e a Taxa Interna de Retorno (TIR) e foram considerados para a avaliação da viabilidade de compra do tomógrafo, sendo que o VPL se mostrou viável apenas para um cenário otimista (VPL >0), com projeção de aumento da demanda de exames em 50\%. Considerando a TIR, esta não é viável em nenhum dos cenários, pois está abaixo da Taxa Mínima de Atratividade (TMA), estabelecida em 6\%. Interessante ressaltar como a TIR e o VPL se complementam apresentado resultados opostos e, mesmo levando em conta o cenário mais positivo, não seria indicado ou viável optar pelo investimento, a não ser que a taxa SELIC diminua neste período do estudo.

\section{REFERÊNCIAS}

BANCO CENTRAL DO BRASIL. BCB. Focus - Relatório de Mercado, 2019. Disponivel em: https://bit.ly/31CNBQy. Acesso em: 12 Agosto 2020.

BRASIL. Sistema Normas Gestão da Informação. Instrução Normativa RFB n 1700 , de 14 de março de 2017, 2017. Disponivel em: https://bit.ly/2VyG0Yv. Acesso em: 23 Agosto 2020. 
BRASIL. Constituição da República Federativa do Brasil de 1988, 2020. Disponivel em: https:// bit.ly/33HmihJ. Acesso em: 23 Agosto 2020.

CASAROTO FILHO, N. Análise de Investimento. 11. ed. São Paulo: Atlas S.A., 2010.

CLEMENTE, A. Projetos Empresariais e Publicos. 3. ed. São Paulo: Atlas S.A., 2008.

DATASUS. Datasus. www.datasus.gov.br, 2019. Acesso em: 20 julho 2019.

FERREIRA, R. G. Engenharia Econônica e Avaliação de Projetos de Investimento. 1. ed. São Pauo - SP: Atlas S.A., 2009.

FGV. FGV. IGP-M varia - 0,67\% em agosto de 2019, 2019. Disponivel em: https://bit.ly/3qqFXfx. Acesso em: 11 de agosto de 2020.

GOLDMAN, P. Viabilidade de Empreendimentos imobiliários: modelagem técnica,orçamento e risco de incorporação. São Paulo: Pini, 2015.

GREER, G. E.; KOLBE, P. T. Investiment Analysis for Real Estate Decision. 5. ed. Estados Unidos: Dearborn Real Estate Education, 2003.

HIRSCHFELD, H. Engenharia econômicca e análise de custos: aplicações práticas para economistas, engenheiros, analistas de investimentos e administradores. São Paulo: Atlas, 2000.

MISHKIN, F. S. Moedas, bancos e mercados financeiros. 5. ed. Rio de Janeiro: LTC, 2000. 474 p.

OLIVIO, R. L. D. F. Análise de Investimento. 1. ed. Campinas: Alinea, 2013.

PEREZ JR, J. H.; DE OLIVEIRA, L. M.; COSTA, R. G. Gestão Estratégica de Custos. 7 ed. São Paulo: Atlas S.A., 2011.

SERTEK, P.; GUINDANI, R. A.; MARTINS, T. S. Administração e Planejamento Estratégico. 20. ed. Curitiba: IBPEX, 2007.

TELES, J. Planejamento e Controle da Produção Descomplicado. 2. ed. Brasília: Engeteles, 2019. 